\title{
Provider confidence in opioid prescribing and chronic pain management: results of the Opioid Therapy Provider Survey
}

This article was published in the following Dove Press journal:

Journal of Pain Research

7 June 2017

Number of times this article has been viewed

\section{Amy CS Pearson' \\ Rajat N Moman² \\ Susan M Moeschler ${ }^{3}$ \\ Jason S Eldrige ${ }^{3}$ \\ W Michael Hooten ${ }^{3}$}

'Department of Anesthesiology \& Perioperative Medicine, Mayo School of Graduate Medical Education, Mayo Clinic, ${ }^{2}$ Mayo Clinic School of Medicine, ${ }^{3}$ Department of Anesthesiology \& Perioperative Medicine, Mayo College of Medicine, Rochester, MN, USA

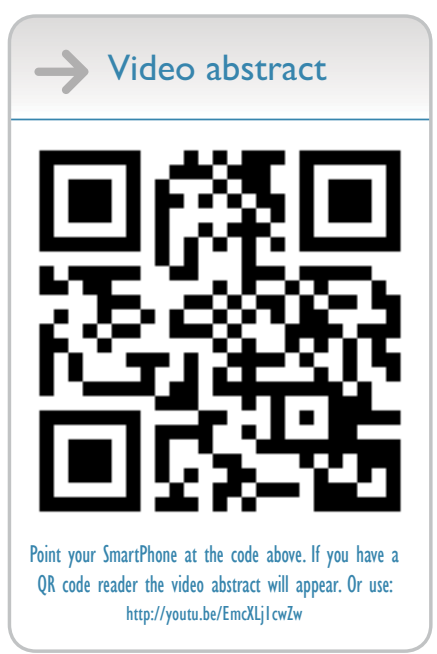

Correspondence: Amy CS Pearson Department of Anesthesiology \& Perioperative Medicine, Mayo Clinic, 200 First St SW, Rochester, MN 55905, USA Tel +l 5072669670

Fax +I 5072667732

Email amy.schultz.pearson@gmail.com
Introduction: Many providers report lack of confidence in managing patients with chronic pain. Thus, the primary aim of this study was to investigate the associations of provider confidence in managing chronic pain with their practice behaviors and demographics.

Materials and methods: The primary outcome measure was the results of the Opioid Therapy Provider Survey, which was administered to clinicians attending a pain-focused continuing medical education conference. Nonparametric correlations were assessed using Spearman's rho. Results: Of the respondents, $55.0 \%$ were women, $92.8 \%$ were white, and $56.5 \%$ were physicians. Primary care providers accounted for $56.5 \%$ of the total respondents. The majority of respondents $(60.8 \%)$ did not feel confident managing patients with chronic pain. Provider confidence in managing chronic pain was positively correlated with 1) following an opioid therapy protocol $(P=0.001), 2)$ the perceived ability to identify patients at risk for opioid misuse $(P=0.006)$, and 3$)$ using a consistent practice-based approach to improve their comfort level with prescribing opioids $(P<0.001)$. Provider confidence was negatively correlated with the perception that treating pain patients was a "problem in my practice" $(P=0.005)$.

Conclusion: In this study, the majority of providers did not feel confident managing chronic pain. However, provider confidence was associated with a protocolized and consistent practicebased approach toward managing opioids and the perceived ability to identify patients at risk for opioid misuse. Future studies should investigate whether provider confidence is associated with measurable competence in managing chronic pain and explore approaches to enhance appropriate levels of confidence in caring for patients with chronic pain.

Keywords: chronic pain, opioids, confidence, continuing medical education

\section{Introduction}

Many providers report lack of confidence in managing patients with chronic pain, even when their patient populations have a high prevalence of chronic pain. ${ }^{1-3}$ Primary care providers report less confidence and satisfaction in dealing with chronic pain than with other common medical problems such as hypertension. ${ }^{3}$ Greater levels of confidence have been associated with increased diagnostic accuracy, advanced provider training, and a greater propensity for information sharing with patients. With regard to pain management, provider confidence in opioid prescribing has been linked to the use of standardized care processes and an increased likelihood of considering pain management a high priority. ${ }^{1,4-7}$

Having an appropriate level of confidence could suggest that a provider has safer opioid-prescribing practices. For this reason, confidence can be considered an important outcome of medical education in regard to opioid-prescribing practices. $^{7-11}$ 
Associations of provider confidence with key parameters of opioid prescribing have not been fully investigated. Thus, the primary aim of this study was to investigate the associations of provider confidence in managing chronic pain with their practice behaviors and demographics.

\section{Materials and methods}

The Mayo Clinic Institutional Review Board (IRB) acknowledged that the activity did not require IRB review based on submitted responses to the IRB application, because the project was designed as a survey for quality improvement purposes. Participation in the survey was voluntary.

\section{Study participants}

The participants in this study were providers attending a course entitled "Mayo Clinic Opioid Conference: Evidence, Clinical Considerations and Best Practice.” Eligible participants included physicians, physician assistants, and nurse practitioners as well as other non-prescribing providers. The survey was offered to 103 attendees of the continuing medical education (CME) course, and $83(80.6 \%)$ took the survey. Fourteen surveys were excluded due to missing data, and the final cohort comprised 69 surveys (67\% completion rate).

\section{Study setting}

The CME conference was held at a conference center in September/October 2016 over the course of 3 days, in which a series of educational 30-minute lectures were delivered from 7:00 am to 12:30 pm. Each lecturer presented information using slides projected on a centrally located screen, and the participants were allowed to ask questions with faculty dialogue after each lecture. Course topics included opioid conversions, comprehensive pharmacology review, unique opioid pharmacokinetics (eg, methadone and buprenorphine), legal concerns, clinical impact of behavioral comorbidities, and review of best practice principles for opioid prescribing, including palliative and addiction considerations. The conference was sponsored by a tertiary referral medical center's CME department, as previously reported. ${ }^{12,13}$

On the first day of the conference, the attendees were asked to complete a survey of their opioid management practices and opinions as well as to provide basic characteristic and demographic information. An audience response system (ARS) was used (Turning Technologies, Youngstown, OH, USA) under the direction of a study investigator (ACSP). Each question was presented on individual slides projected on a main screen. Participants electronically provided their answers on the ARS devices via a lettered keypad. The result was transmitted wirelessly to a central computer and downloaded as a spreadsheet of raw data. Data were linked to individual devices, which were not assigned to specific participants. On completion of the survey, participants were offered a small $(<\$ 10)$ gift for their participation.

\section{Data collection}

\section{Demographics and practice characteristics}

The demographic data collected included age, sex, race, years in practice, practice setting, hours worked/week, number of weekly patient encounters, and previous training in pain management.

\section{Opioid Therapy Provider Survey (OTS)}

The primary outcome measure was the results of OTS. It is a ten-question survey first reported by Jamison et al, ${ }^{2,9}$ as a means of assessing providers' practice behaviors and confidence in managing patients on opioid therapy. The ten items are "Treating patients with pain is a problem in my practice," "I am willing to prescribe opioids with support from a pain clinic," "I fear my patients will become addicted to opioids," "I am dissatisfied with communication with pain specialists," "I follow a recommended opioid therapy protocol," "I would likely prescribe opioids when other treatments are ineffective," "I can identify patients at risk for misuse of pain medication," "I am confident in my ability to manage patients with chronic pain," "I am satisfied with transition notes from pain clinics," and "The consistent approach of my practice has helped me feel comfortable in prescribing opioids." The survey is scored on a 5-point Likert scale, where 1= strongly agree and $5=$ strongly disagree. This study was developed by experienced researchers after an extensive review of the literature, and reliability and validity have been established., ${ }^{2,9}$

\section{Statistical analysis}

Demographic and practice characteristic data were reported, and the response frequency to each item was analyzed. Nonparametric correlations were assessed comparing the statement "I am confident in my ability to manage patients with chronic pain" with the nine other statements of OTS using Spearman's rho. This technique was repeated comparing the statement "I am confident in my ability to manage patients with chronic pain" with the demographic parameters using Spearman's rho. Two-sided tests were used in all analyses, and the level of significance for all statistical tests was set at $P<0.05$. All the analyses were carried out using IBM SPSS Statistics for Windows, Version 21.0 (IBM Corporation, Armonk, NY, USA; released 2012). 


\section{Results}

\section{Demographics and practice characteristics}

The demographic and practice characteristics are provided in Table 1 and include age, sex, race, years in practice, practice setting, hours worked/ week, number of weekly patient encounters, and previous training in pain management.

Fifty-five percent of the respondents were women, $92.8 \%$ were white, and $56.5 \%$ were physicians. The majority practiced in small cities (39.1\%) or rural areas (26.1\%). Forty-two percent worked in private practice, and $56.5 \%$ were primary care providers. Regarding postgraduate training in pain management, $44.9 \%$ reported $>8$ hours of additional pain management training, and $34.8 \%$ had a state requirement for opioid-related continuing education.

Table I Participant demographics

\begin{tabular}{ll}
\hline Characteristics & Total number of \\
& participants, $\mathbf{n}$ (\%) \\
\hline Age (years) & \\
$25-35$ & $7(10.1)$ \\
$36-44$ & $19(27.5)$ \\
$45-54$ & $10(14.5)$ \\
$55-64$ & $21(30.4)$ \\
$\geq 65$ & $9(13.0)$ \\
Sex & \\
Female & $38(55.1)$ \\
Male & $27(39.1)$ \\
Years in practice & \\
$0-5$ & $8(11.6)$ \\
$6-10$ & $7(10.1)$ \\
II-15 & $9(13.0)$ \\
I6-20 & $11(15.9)$ \\
$21-25$ & $7(10.1)$ \\
$26-30$ & $10(14.5)$ \\
$\geq 31$ & $15(21.7)$ \\
Race/ethnicity & \\
African American/Black & $0(0)$ \\
Hispanic/Latino & $2(2.9)$ \\
Asian & $2(2.9)$ \\
Caucasian/White & $64(92.8)$ \\
Number of patients seen weekly & $18(26.1)$ \\
$0-19$ & $5(7.2)$ \\
$20-39$ & $6(8.7)$ \\
$40-59$ & $18(26.1)$ \\
$60-79$ & $15(21.7)$ \\
$80-99$ & $15(21.7)$ \\
$\geq 100$ & $8(11.6)$ \\
Practice location & $12(17.4)$ \\
Urban & $10(14.5)$ \\
Suburban & $27(39.1)$ \\
Small city & \\
Rural & \\
\hline & \\
&
\end{tabular}

(Continued)
Table I (Continued)

\begin{tabular}{ll}
\hline Characteristics & $\begin{array}{l}\text { Total number of } \\
\text { participants, } \mathbf{n} \text { (\%) }\end{array}$ \\
\hline Primary work facility & $29(42.0)$ \\
Private practice & $13(18.8)$ \\
Academic center & $9(13.0)$ \\
Community health center & $0(0)$ \\
Government facility & $2(2.9)$ \\
Long-term care facility & $3(4.3)$ \\
Locum tenens & $10(14.5)$ \\
Other & \\
Previous additional training in pain & \\
management & $9(13.0)$ \\
Yes, 0-8 hours & $31(44.9)$ \\
Yes, >8 hours & $27(39.1)$ \\
No & \\
State requirement for opioid-related & \\
continuing education & $24(34.8)$ \\
Yes & \\
Primary care provider & $39(56.5)$ \\
Yes & $28(40.6)$ \\
No & \\
Typical number of hours worked/week & $0(0)$ \\
$<20$ & $9(13.0)$ \\
$20-39$ & $25(36.2)$ \\
$40-49$ & $21(30.4)$ \\
$50-59$ & $12(17.4)$ \\
$\geq 60$ & $18(26.1)$ \\
Provider type & $8(11.6)$ \\
Advanced practice nurse & $39(56.5)$ \\
Physician assistant & $1(1.4)$ \\
Physician & $2(2.9)$ \\
Registered nurse & \\
Other & \\
\hline
\end{tabular}

\section{The OTS results}

A majority of the participants agreed or strongly agreed with the statement (abbreviated) "Treating pain patients is a problem in my practice" (60.8\%) (Table 2). Regarding opioid management practice patterns, participants also agreed or strongly agreed with the following statements: 1) "I am willing to prescribe opioids with support from a pain clinic" (71.0\%); 2) "I would likely prescribe opioids when other treatments are ineffective" (79.7\%); 3) "I can identify patients at risk for misuse of pain medication" (53.6\%); and 4) "The consistent approach of my practice has helped me feel comfortable in prescribing opioids" $(60.9 \%)$. The majority also agreed or strongly agreed with "I fear my patients will become addicted to opioids" (55.1\%).

A majority of the participants were neutral to, disagreed with, or strongly disagreed with "I follow a recommended opioid therapy protocol" (55.0\%), "I am confident in my ability to manage patients with chronic pain" (60.8\%), and "I am satisfied with transition notes from the pain center" (84.0\%). 
Table 2 Responses to Opioid Therapy Provider Survey questions ${ }^{\mathrm{a}}$

\begin{tabular}{|c|c|c|c|c|c|c|}
\hline Opioid Therapy Provider Survey question ${ }^{a}$ & $\begin{array}{l}\text { Strongly } \\
\text { agree }\end{array}$ & Agree & Neutral & Disagree & $\begin{array}{l}\text { Strongly } \\
\text { disagree }\end{array}$ & $\begin{array}{l}\text { No } \\
\text { answer }\end{array}$ \\
\hline Treating pain patients is a problem in my practice & $9(13)$ & $33(47.8)$ & $14(20.3)$ & $7(10.1)$ & $2(2.9)$ & $4(5.8)$ \\
\hline I am willing to prescribe opioids with support from pain clinic & $\mathrm{II}(15.9)$ & $38(55.1)$ & $15(21.7)$ & $2(2.9)$ & $0(0)$ & $3(4.3)$ \\
\hline I fear my patients will become addicted to opioids & $4(5.8)$ & $34(49.3)$ & $18(26.1)$ & II (I5.9) & $0(0)$ & $2(2.9)$ \\
\hline I am dissatisfied with communication with pain specialists & $10(14.5)$ & $23(33.3)$ & $20(29.0)$ & $10(14.5)$ & $5(7.2)$ & I (I.4) \\
\hline I follow a recommended opioid therapy protocol & $8(11.6)$ & $23(33.3)$ & $19(27.5)$ & II (I5.9) & $4(5.8)$ & $4(5.8)$ \\
\hline I would likely prescribe opioids when other treatments are ineffective & $12(17.4)$ & $43(62.3)$ & $8(11.6)$ & $4(5.8)$ & $0(0)$ & $2(2.9)$ \\
\hline I can identify patients at risk for misuse of pain medication & I ( 1.4$)$ & $36(52.2)$ & $16(23.2)$ & $13(18.8)$ & $0(0)$ & $3(4.3)$ \\
\hline I am confident of my ability to manage patients with chronic pain & $3(4.3)$ & $24(34.8)$ & $18(26.1)$ & $17(24.6)$ & $5(7.2)$ & $2(2.9)$ \\
\hline I am satisfied with the transition notes from the pain center & $3(4.3)$ & $8(11.6)$ & $26(37.7)$ & $25(36.2)$ & $6(8.7)$ & I (I.4) \\
\hline $\begin{array}{l}\text { The consistent approach of my practice has helped me feel comfortable in } \\
\text { prescribing opioids }\end{array}$ & $6(8.7)$ & $36(52.2)$ & $17(24.6)$ & $7(10.1)$ & $3(4.3)$ & $0(0.0)$ \\
\hline
\end{tabular}

Notes: Questions are truncated from original version in this report. ${ }^{a}$ Values in parentheses are reported as $\mathrm{n}(\%)$. Percentages may not equal I00 due to rounding.

Table 3 Correlation between responses to survey questions and provider confidence

\begin{tabular}{lll}
\hline Survey response & Spearman's coefficient & P-value \\
\hline Treating pain patients is a problem in my practice & -0.349 & 0.005 \\
I am willing to prescribe opioids with support from a pain clinic & -0.067 & 0.594 \\
I fear my patients will become addicted to opioids & -0.23 I & 0.064 \\
I am dissatisfied with communication with pain specialists & -0.067 & 0.398 \\
I follow a recommended opioid therapy protocol & 0.146 & 0.00 I \\
I would likely prescribe opioids when other treatments are ineffective & 0.339 & 0.245 \\
I can identify patients at risk for misuse of pain medication & 0.206 & 0.006 \\
I am satisfied with transition notes from pain clinics & 0.445 & 0.097 \\
The consistent approach of my practice has helped me feel comfortable in prescribing opioids & $<0.000$ I \\
\hline
\end{tabular}

\section{Provider confidence with chronic pain management}

Table 3 shows the correlation of the responses to the statement "I am confident in my ability to manage patients with chronic pain" with the remaining statements of OTS. Provider confidence was positively correlated with the following three statements: 1) "I follow a recommended opioid therapy protocol" $(P=0.001) ; 2)$ "I can identify patients at risk for misuse of pain medications" ( $P=0.006)$; and 3) "The consistent approach of my practice has helped me feel comfortable in prescribing opioids" $(P<0.0001)$. Provider confidence was negatively correlated with the following statement: "Treating pain patients is a problem in my practice" $(P=0.005)$.

No significant correlations were observed between provider confidence and the five following statements: 1) "I am willing to prescribe opioids with support from a pain clinic"; 2) "I fear my patients will become addicted to opioids"; 3) "I am dissatisfied with communication with pain specialists"; 4) "I would likely prescribe opioids when other treatments are ineffective"; and 5) "I am satisfied with transition notes from pain clinics." No significant correlations were observed between provider confidence and any of the reported demographic variables (Table 4).
Table 4 Correlation between provider demographics and provider confidence

\begin{tabular}{lll}
\hline Demographics & $\begin{array}{l}\text { Spearman's } \\
\text { coefficient }\end{array}$ & P-value \\
\hline Age & -0.030 & 0.813 \\
Sex & 0.066 & 0.609 \\
Years in practice & -0.163 & 0.194 \\
Race & -0.030 & 0.813 \\
Average number of patients & 0.162 & 0.197 \\
Practice location & 0.044 & 0.726 \\
Type of practice & -0.156 & 0.219 \\
Additional training in pain management & 0.201 & 0.108 \\
Required opioid-related continuing education & 0.005 & 0.966 \\
Primary care provider & 0.010 & 0.935 \\
Average hours worked & -0.034 & 0.791 \\
Type of provider & -0.076 & 0.543 \\
\hline
\end{tabular}

\section{Discussion}

In this study, most providers $(60.8 \%)$ were not confident of their ability to manage chronic pain. This finding is consistent with much of the medical literature regarding provider confidence in opioid prescribing. Jamison et al also used OTS to assess providers' confidence in prescribing opioids. ${ }^{2,9}$ Not only were providers not confident of their ability to manage patients with chronic pain initially $(42.9 \%)$, but their levels 
of confidence 1 year after targeted intervention did not significantly improve $(44.7 \%, P>0.05)$. However, the providers' confidence in specifically prescribing opioids for non-cancer pain did significantly improve with intervention $(P=0.001){ }^{2}$

In this study, the providers who reported confidence in managing chronic pain were also likely to report that they used a consistent approach in prescribing opioids and that they followed a recommended opioid therapy protocol. This finding has also been demonstrated by Alford et al, who delivered a 3-hour opioid training module to 2,850 clinicians. ${ }^{8}$ Two months after the intervention, the majority of responding providers reported increased confidence in communication with patients, even when the treatment showed no benefit, as well as increased confidence in monitoring of ongoing therapy, and their assessment of patient risk/benefit and misuse potential. The majority also improved their adherence to recommended best practices surrounding urine drug testing, documentation, and patient education, and sustained improvements in medical knowledge at 2 months.

It was found that clinician confidence in opioid prescribing was also correlated with confidence in identifying patients at risk for misuse, but not with fear of addiction. This is consistent with previous studies, which found that primary care providers (PCPs) who were confident of prescribing opioids were less concerned about the negative consequences of opioid prescribing, including risk of addiction. ${ }^{7,14,15}$ Wenghofer et al surveyed 658 PCPs and found that physicians who reported confidence in prescribing opioids were more likely to believe in the pain-relieving and functional benefits of opioids and less likely to believe that many patients become addicted to opioids. ${ }^{15}$

The correlation between provider confidence in managing chronic pain and age, sex, race, provider type, years in practice, practice facility/location, or number of weekly patient encounters was not found. This is consistent with the findings of Lum et al, who found that providers' confidence in prescribing opioids to a specific population with chronic pain was not associated with profession, age, or race. ${ }^{1}$ Other studies have also reported that provider demographics do not correlate with the level of confidence; although some have found that male gender and increased age are associated with higher levels of confidence. , $^{1,9,16}$

It was also found that additional post-training education in pain management and presence of state opioid-related CME requirements were not correlated with any responses on OTS. This is inconsistent with O'Rorke et al's finding that postresidency education was correlated with an increased level of physician comfort in caring for patients with chronic pain. ${ }^{17}$
To the authors' knowledge, there have been no formal studies on the effect of state-required CME on providers' opioid knowledge, attitudes, or prescribing patterns. It is possible that more frequent, targeted, and practical opioid education interventions than are currently available may prove more effective in improving providers' knowledge, attitudes, and beliefs toward opioid prescribing.

While the degree of overconfidence in opioid prescribing and addiction risk assessment was not measured in this study, it should be considered that overconfidence may be associated with risky opioid management. In a survey study of 658 PCPs, $86 \%$ reported being confident about prescribing opioids, even though $42 \%$ reported that at least one patient had experienced an opioid-related adverse event in the past years. ${ }^{15}$ Another survey of PCPs found that the majority were likely to prescribe opioids to a patient with active substance abuse. ${ }^{3}$ There is evidence that practices such as considering alternatives, broadening information-gathering options, fostering a supportive work environment, and providing real-time feedback may be useful tools in correcting overconfidence. $^{18}$

\section{Limitations}

This study has limitations. The respondents were providers attending an opioid prescribing conference, who were potentially more motivated to improve their opioid prescribing compared to the general population of all opioid prescribers. Also, this study did not evaluate providers' actual competence in opioid prescribing in chronic pain management. While confidence may be associated with improved outcomes in patient education and diagnostic accuracy, it may also be associated with false confidence or overconfidence, which can produce poorer patient outcomes. ${ }^{4,19,20}$ It is unclear from this study whether providers' confidence or lack of confidence was consistent with their knowledge and practices. Future studies should address the correlations between provider confidence and clinical competence as measured by providers' knowledge, satisfaction, and outcomes.

\section{Conclusion}

In this study, the majority of providers were not confident of their ability to manage chronic pain when surveyed prior to a comprehensive opioid-related CME event. While confidence was associated with using a consistent approach to practice and following a recommended prescribing protocol, it was also associated with a lower concern for the addictive effects of opioids. Future studies, using a repeated measures design, could better delineate whether provider confidence 
is associated with measurable competence and explore approaches to correcting overconfidence in opioid prescribing and risk stratification. The effectiveness of continuing education courses should also be evaluated, as they could provide a means for improving providers' knowledge and attitudes toward opioid prescribing and chronic pain management.

\section{Acknowledgment}

The authors thank Sierra Tollefson and Gregory Brandt for their assistance in the implementation of the survey.

\section{Disclosure}

The authors report no conflicts of interest in this work.

\section{References}

1. Lum PJ, Little S, Botsko M, et al. Opioid-prescribing practices and provider confidence recognizing opioid analgesic abuse in HIV primary care settings. J Acquir Immune Defic Syndr. 2011;56(Suppl 1): S91-S97.

2. Jamison RN, Scanlan E, Matthews ML, Jurcik DC, Ross EL. Attitudes of primary care practitioners in managing chronic pain patients prescribed opioids for pain: a prospective longitudinal controlled trial. Pain Med. 2016;17(1):99-113.

3. Vijayaraghavan M, Penko J, Guzman D, Miaskowski C, Kushel MB. Primary care providers' views on chronic pain management among high-risk patients in safety net settings. Pain Med. 2012;13(9): 1141-1148.

4. Ishikawa H, Son D, Eto M, Kitamura K, Kiuchi T. The informationgiving skills of resident physicians: relationships with confidence and simulated patient satisfaction. BMC Med Educ. 2017;17(1):34.

5. Uy RC, Sarmiento RF, Gavino A, Fontelo P. Confidence and information access in clinical decision-making: an examination of the cognitive processes that affect the information-seeking behavior of physicians. AMIA Annu Symp Proc. 2014;2014:1134-1140.

6. Yazbek J, Ameye L, Testa AC, et al. Confidence of expert ultrasound operators in making a diagnosis of adnexal tumor: effect on diagnostic accuracy and interobserver agreement. Ultrasound Obstet Gynecol. 2010;35(1):89-93.
7. Macerollo AA, Mack DO, Oza R, Bennett IM, Wallace LS. Academic family medicine physicians' confidence and comfort with opioid analgesic prescribing for patients with chronic nonmalignant pain. J Opioid Manag. 2014;10(4):255-261.

8. Alford DP, Zisblatt L, Ng P, et al. SCOPE of pain: an evaluation of an opioid risk evaluation and mitigation strategy continuing education program. Pain Med. 2016;17(1):52-63.

9. Jamison RN, Sheehan KA, Scanlan E, Matthews M, Ross EL. Beliefs and attitudes about opioid prescribing and chronic pain management: survey of primary care providers. J Opioid Manag. 2014;10(6):375-382.

10. Lalonde L, Leroux-Lapointe V, Choiniere M, et al. Knowledge, attitudes and beliefs about chronic noncancer pain in primary care: a Canadian survey of physicians and pharmacists. Pain Res Manag. 2014;19(5): 241-250.

11. Regunath $\mathrm{H}$, Cochran $\mathrm{K}$, Cornell $\mathrm{K}$, et al. Is it painful to manage chronic pain? A cross-sectional study of physicians in-training in a university program. Mo Med. 2016;113(1):72-78.

12. Hooten WM, Bruce BK. Beliefs and attitudes about prescribing opioids among healthcare providers seeking continuing medical education. J Opioid Manag. 2011;7(6):417-424.

13. Pearson AC, Eldrige JS, Moeschler SM, Hooten WM. Opioids for chronic pain: a knowledge assessment of nonpain specialty providers. J Pain Res. 2016;9:129-135.

14. Potter M, Schafer S, Gonzalez-Mendez E, et al. Opioids for chronic nonmalignant pain. Attitudes and practices of primary care physicians in the UCSF/Stanford Collaborative Research Network. University of California, San Francisco. J Fam Pract. 2001;50(2):145-151.

15. Wenghofer EF, Wilson L, Kahan M, et al. Survey of Ontario primary care physicians' experiences with opioid prescribing. Can Fam Physician. 2011;57(3):324-332.

16. Starrels JL, Fox AD, Kunins HV, Cunningham CO. They don't know what they don't know: internal medicine residents' knowledge and confidence in urine drug test interpretation for patients with chronic pain. J Gen Intern Med. 2012;27(11):1521-1527.

17. O'Rorke JE, Chen I, Genao I, Panda M, Cykert S. Physicians' comfort in caring for patients with chronic nonmalignant pain. Am J Med Sci. 2007;333(2):93-100.

18. Meyer AN, Singh H. Calibrating how doctors think and seek information to minimise errors in diagnosis. BMJ Qual Saf. 2016.

19. Saposnik G, Redelmeier D, Ruff CC, Tobler PN. Cognitive biases associated with medical decisions: a systematic review. BMC Med Inform Decis Mak. 2016;16(1):138.

20. Berner ES, Graber ML. Overconfidence as a cause of diagnostic error in medicine. Am J Med. 2008;121(5 Suppl):S2-S23.
Journal of Pain Research

Publish your work in this journal

The Journal of Pain Research is an international, peer reviewed, open access, online journal that welcomes laboratory and clinical findings in the fields of pain research and the prevention and management of pain. Original research, reviews, symposium reports, hypothesis formation and commentaries are all considered for publication.

\section{Dovepress}

The manuscript management system is completely online and includes a very quick and fair peer-review system, which is all easy to use. Visit http://www.dovepress.com/testimonials.php to read real quotes from published authors. 